\title{
Antikörper verbessert C-Peptid-Antwort
}

Fragestellung: Kann Teplizumab den voranschreitenden CPeptidabfall bei Typ-1-Diabetikern verhindern, wenn es nach der Manifestationsperiode gegeben wird?

Hintergrund: Der Typ-1-Diabetes resultiert aus einem chronischen autoimmunen Prozess, der sich über Jahre hinzieht und auch nach der Diagnose noch weitergeht. Teplizumab (ein nicht an den Fc-Rezeptor bindender anti-CD3 monoklonaler Antikörper) könnte diesen Prozess beeinflussen.

Patienten und Methodik: In dieser randomisierten Studie erhielten 58 Teilnehmer, bei denen der Typ-1-Diabetes schon 4 bis 12 Monate bestand, Teplizumab oder Placebo. Die multizentrische Studie wurde an vier akademischen Einrichtungen in den USA realisiert. In einem zentralen Randomisierungzentrum teilte man mithilfe von Computer-generierten Tabellen die Behandlungen zu. Untersucher, Patienten und die Zentren waren verblindet. Primärer Endpunkt war der Vergleich der MixedMeal-stimulierten C-Peptidantwort nach einem Jahr. Exploratisch untersuchten die Autoren Subgruppen.

Ergebnisse: 34 Personen wurden der Teplizumab- und 29 der Placebo-Gruppe zugeteilt. Die Autoren analysierten Daten von 31 bzw. 27 Personen. Die Analyse des primären Endpunktes ergab, dass nach 12 Monaten eine 21,7\% höhere C-Peptidantwort in der Teplizumab-Gruppe (0,45 vs. 0,371; Unterschied, 0,59 [95\%-KI: 0,06, 0,115] nmol/l, $\mathrm{p}=0,03$ ) auftrat als in der PlaceboGruppe. Nach Korrektur auf initiale $\mathrm{HbA}_{1 \mathrm{c}}$-Werte zeigte sich, dass die C-Peptidwerte in der Teplizumab-Gruppe 17,7\% höher $(0,44$ vs. 0,378 ; Unterschied, 0,049 [95\%-KI $0,0,108] \mathrm{nmol} / \mathrm{l}$, $\mathrm{p}=0,09)$ waren. Bei einem größeren Anteil der Placebo behandelten Probanden sank das messbare C-Peptid nach 12 Monaten $(\mathrm{p}=0,03)$. Die Teplizumab-Gruppe benötigte weniger exogenes Insulin $(\mathrm{p}<0,001)$. Die $\mathrm{HbA}_{1 c}$-Werte waren nicht unter-

\section{-Kommentar von PD Dr. med. Nanette C. Schloot}

\section{Das macht Mut, weiter zu forschen}

Diese interessante Studie wurde aus Drittmittelgeldern finanziert und zeigt, dass es sich lohnt, "negativ publizierte" Daten zu einer Substanz nicht aufzugeben. Im Jahr 2011 hatte die Protege-Studie ja gezeigt, dass der primäre kombinierte Endpunkt aus $\mathrm{HbA}_{1 \mathrm{c}}$ und Insulinbedarf sich bei neumanifesten Typ1-Diabetikern mit Teplizumab nicht ausreichend verbessert. Unteranalysen hatten jedoch auch damals schon einen günstigen Effekt auf das C-Peptid ergeben. Probleme wie die schlechte Blutglukoseeinstellung der indischen Patienten lieBen damals die untersuchte Gesamtgruppe sehr heterogen werden. Die aktuelle Studie von der Herold-Gruppe ergab nun, dass Teplizumab doch wirken kann. Auch wenn die beobachteten Effekte weit entfernt von einer tatsächlichen Heilung sind, machen sie Mut, in diesem Bereich weiter zu forschen.

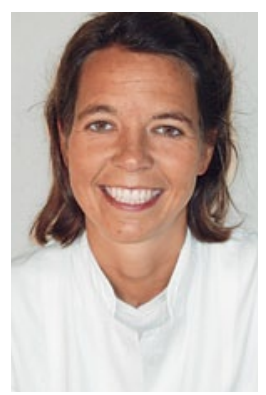

PD Dr. med. Nanette C. Schloot

schiedlich. Teplizumab wurde gut vertragen. In der Subgruppenanalyse war der Behandlungsvorteil bei jungen Menschen und bei Personen mit einem $\mathrm{HbA}_{1 c}<6,5 \%$ zu Studienbeginn größer.

Die zirkulierenden CD8 zentralen Gedächtnis-T-Zellen von klinischen Teplizumab-Respondern stiegen zwei Monate nach Therapiebeginn im Vergleich zu den Non-Respondern.

Schlussfolgerungen: Diese Studie lässt vermuten, dass eine Immuntherapie mit Teplizumab die endogene Insulinsekretion günstig beeinflusst, auch wenn sie nach der Manifestationsperiode gegeben wird. Allerdings ist das Ausmaß des Therapieerfolgs geringer als bei Therapie in der Manifestationsperiode. Die in dieser Studie definierten Patientencharakteristika zeigen, wer am ehesten für eine solche Immuntherapie geeignet ist.
Herold KC, Gitelman SE, Willi SM et al. Teplizumab treatment may improve C-peptide responses in participants with type 1 diabetes after the new-onset period: a randomised controlled trial. Diabetologia 2013;56(2): 391-400.

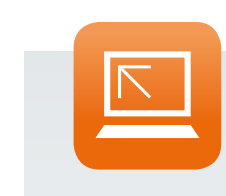

Weitere Beiträge im Dossier zum Thema „Endokrinologie" finden Sie online unter $>$ www. springerinnereendokrinologie

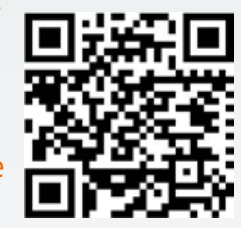
medizin.de/
Gastwissenschaftlerin

Deutsches Diabetes-Zentrum an der Heinrich-Heine Universität Düsseldorf Institut für Klinische Diabetologie Auf'm Hennekamp 54 40225 Düsseldorf schloot@ddz.uni-duesseldorf.de 